11

\title{
Нелинейное затухание и нелинейный фазовый набег интенсивных спиновых волн в экранированных ферритовых пленках
}

\author{
(C) А.Э. Козин, ${ }^{1,2}$ А.Б. УСтинов ${ }^{1}$ \\ ${ }^{1}$ Санкт-Петербургский государственный электротехнический университет „ЛЭТИ“, \\ 197376 Санкт-Петербург, Россия \\ ${ }^{2}$ ОАО „Завод Магнетон“, \\ 194223 Санкт-Петербург, Россия \\ e-mail: ustinov-rus@mail.ru
}

(Поступило в Редакцию 25 апреля 2018 г.)

\begin{abstract}
Впервые изучены нелинейное затухание и нелинейный фазовый набег интенсивных поверхностных спиновых волн в экранированных пленках железо-иттриевого граната. Определены коэффициенты нелинейного затухания феноменологической модели распространения нелинейных спиновых волн в таких пленках. Обнаружено, что нелинейный фазовый набег в экранированных пленках является слабым эффектом по сравнению со свободными пленками и составляет десятки градусов. В то же время нелинейное затухание в экранированных пленках сопоставимо по величине со свободными пленками. Показано, что для пленок микронной толщины при увеличении мощности до $15 \mathrm{dBm}$ вносимые потери возрастают на $2-5 \mathrm{~dB}$.
\end{abstract}

DOI: 10.21883/JTF.2019.02.47085.164-18

Спиновые волны (CB), распространяющиеся в эпитаксиальных ферритовых пленках, имеют широкие возможности применения для обработки и генерации сверхвысокочастотных (СВЧ) сигналов. Разнообразие дисперсионных и нелинейных свойств СВ позволили создать различные СВЧ-приборы, такие как фильтры, линии задержки, фазовращатели, шумоподавители и ограничители мощности [1-3]. Для возбуждения СВ обычно используются антенны, выполненные в виде отрезков одиночных и многоэлементных микрополосковых линий, щелевых линий, копланарных волноводов, а также их комбинаций $[4,5]$.

В последние годы возрос интерес к применению нелинейных свойств СВ для создания приборов обработки СВЧ-сигналов. Так, были разработаны нелинейные фазовращатели [6-8], интерферометры [9,10], направленные ответвители $[11,12]$, а также генераторы стационарных и хаотических сигналов $[13,14]$.

Принцип действия перечисленных выше устройств основан на эффекте нелинейного фазового набега интенсивных СВ. В основе этого эффекта лежит процесс нелинейного сдвига собственных частот интенсивных СВ при изменении их амплитуды. В свою очередь причиной нелинейного сдвига собственных частот является уменьшение постоянной составляющей намагниченности при увеличении амплитуды СВ. Как следует из модели прецессии магнитного момента [2], увеличение переменной компоненты намагниченности приводит к уменьшению постоянной компоненты намагниченности. Постоянная намагниченность входит в качестве параметра в дисперсионное уравнение $\mathrm{CB}$, и ее изменение приводит к смещению дисперсионной кривой. В результате на определенной частоте происходит изменение волнового числа $\mathrm{CB}$, а следовательно, и фазы $\mathrm{CB}$ на выходе устройства.
На величину нелинейного фазового набега влияет нелинейное затухание СВ $[2,6,12,15]$. Нелинейное затухание СВ связано с развитием четырехволнового процесса рассеяния основной волны на волны, далеко отстоящие от нее по частоте. К настоящему моменту времени такие нелинейные процессы достаточно хорошо изучены в неэкранированных пленках. В экранированных пленках они до сих пор не изучались.

Целью настоящей работы является экспериментальное и теоретическое исследование нелинейного затухания и нелинейного фазового набега поверхностных СВ в ферритовых пленках, вблизи поверхности которых расположен металлический экран.

Экспериментальные исследования проводились на макете (рис. 1), представлявшем собой линию задержки на основе пленки железо-иттриевого граната (ЖИГ). В эксперименте использовались волноводы СВ 1, из-

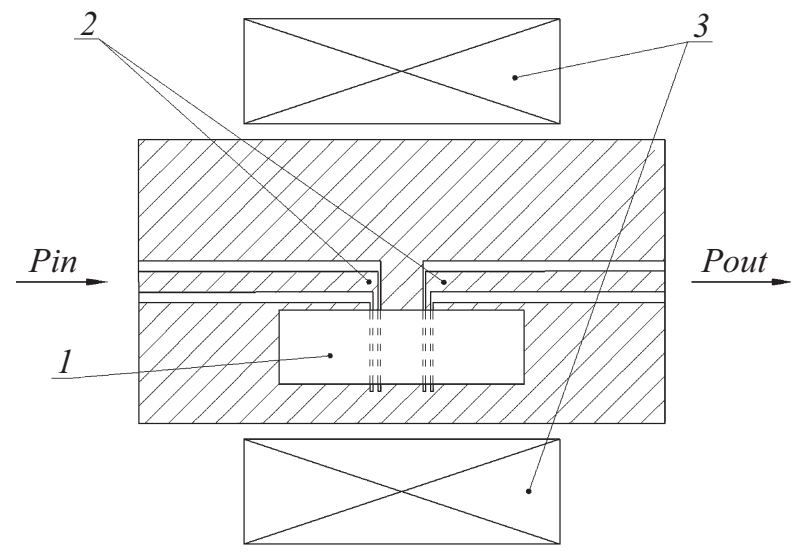

Рис. 1. Блок-схема экспериментального макета: 1 - волноводы СВ, 2 - копланарные короткозамкнутые антенны, 3 электромагнит. 

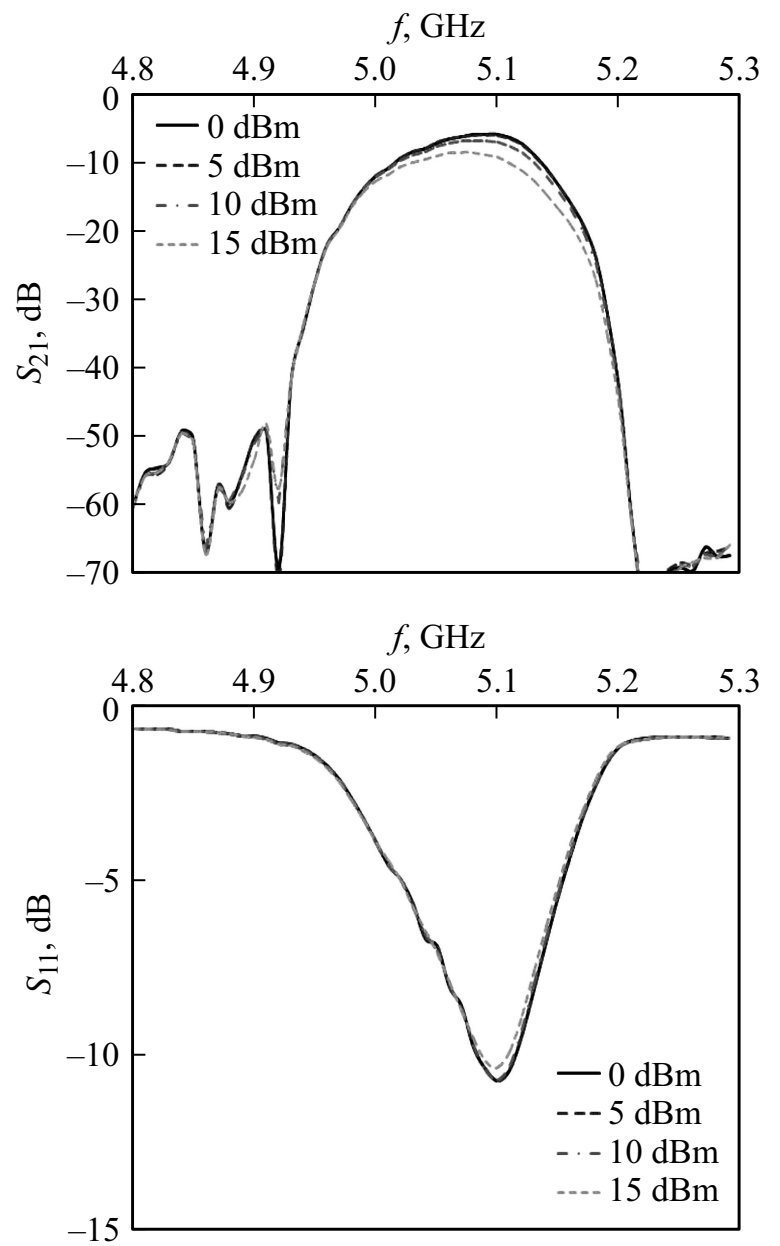

$b$
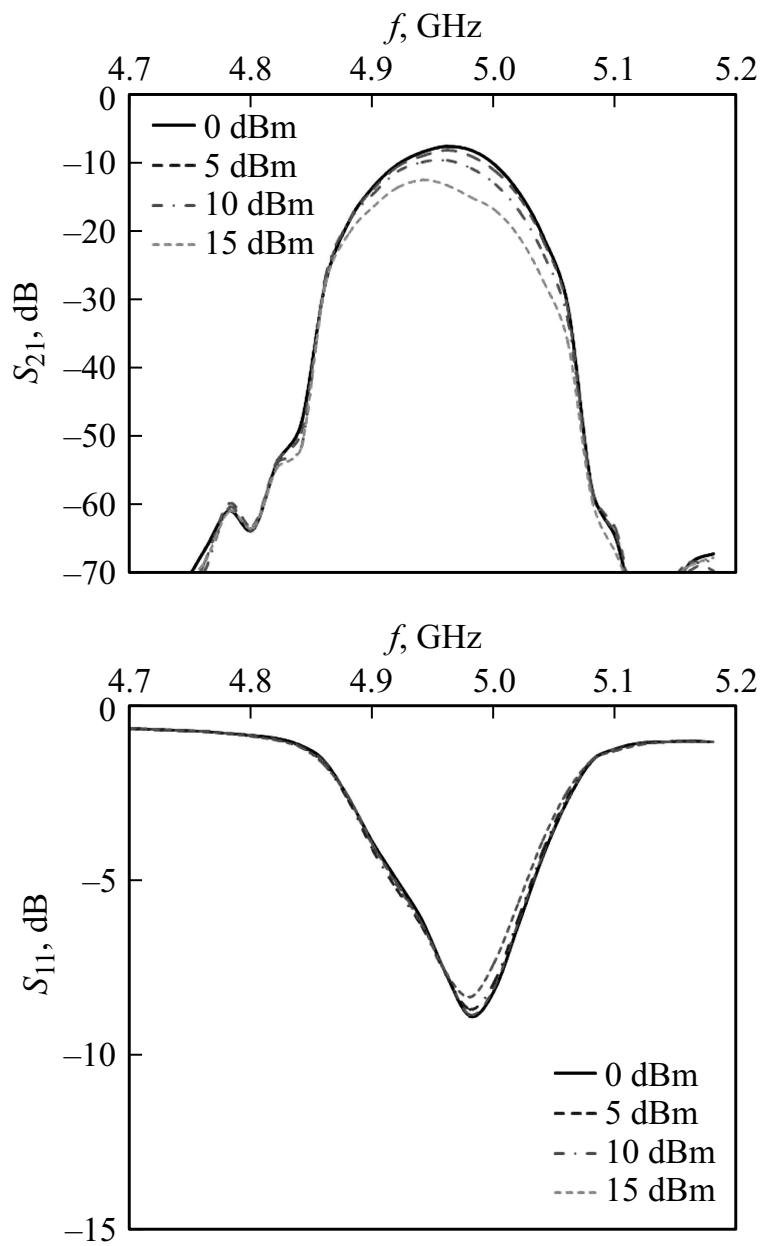

Рис. 2. Вносимые потери (сверху) и обратные потери (снизу) в зависимости от частоты, измеренные при различных мощностях СВЧ-сигнала для пленок ЖИГ толщинами: $L_{1}=5.2 \mu \mathrm{m}(a) ; L_{2}=3.1 \mu \mathrm{m}(b)$.

готовленные из эпитаксиальных пленок ЖИГ двух толщин - $L_{1}=5.2 \mu \mathrm{m}$ и $L_{2}=3.1 \mu \mathrm{m}$ с намагниченностью насыщения $4 \pi M_{S}=1750 \mathrm{Gs}$ и линейным параметром диссипации около $\Delta H=0.5 \mathrm{Oе}$. Ширина волноводов была $3 \mathrm{~mm}$, длина $30 \mathrm{~mm}$. Для возбуждения и приема СВ использовались копланарные короткозамкнутые антенны 2 с шириной центрального проводника $100 \mu \mathrm{m}$ и шириной щелей $50 \mu \mathrm{m}$. Расстояние между антеннами $d$ составляло $4 \mathrm{~mm}$, а длина антенн равнялась $3 \mathrm{~mm}$. Подвод СВЧ-мощности к антеннам осуществлялся копланарными линиями с волноводным сопротивлением $50 \Omega$. Антенны и подводящие линии были выполнены методом фотолитографии на подложке из поликора толщиной $0.5 \mathrm{~mm}$. Макет помещался в постоянное магнитное поле электромагнита 3, направленное в плоскости пленки параллельно антеннам и перпендикулярно направлению распространения СВ. Такая взаимная ориентация постоянного магнитного поля и пленки соответствует распространению в ней поверхностных СВ. Значение напряженности магнитного поля $H_{0}$ составляло около 1050 Oе.
Необходимо отметить две существенные особенности экспериментального макета. Во-первых, при использовании копланарных антенн одна из сторон пленки оказывается экранированной металлической плоскостью, что приводит к увеличению групповой скорости СВ и расширению области их возбуждения. Во-вторых, поверхностные СВ хорошо „чувствуют“ близость экрана у поверхности пленки в силу экспоненциального распределения амплитуды переменной намагниченности по толщине пленки с максимумом на ее поверхности [16].

В ходе исследований измерялись $S$-параметры экспериментального макета на векторном анализаторе цепей R\&S ZVB 20. Частотные зависимости вносимых и обратных потерь макета, измеренные при различных уровнях мощности СВЧ-сигнала, приведены на рис. 2. Из графиков видно, что полоса пропускания устройства составляла около $250 \mathrm{MHz}$ по уровню минус $50 \mathrm{~dB}$ с центральной частотой $5050 \mathrm{MHz}$ для волновода толщиной $L_{1}$ и $4950 \mathrm{MHz}$ для волновода толщиной $L_{2}$. Вносимые потери на центральной частоте полосы пропускания при сравнительно низком уровне входной мощности $0 \mathrm{dBm}$ 

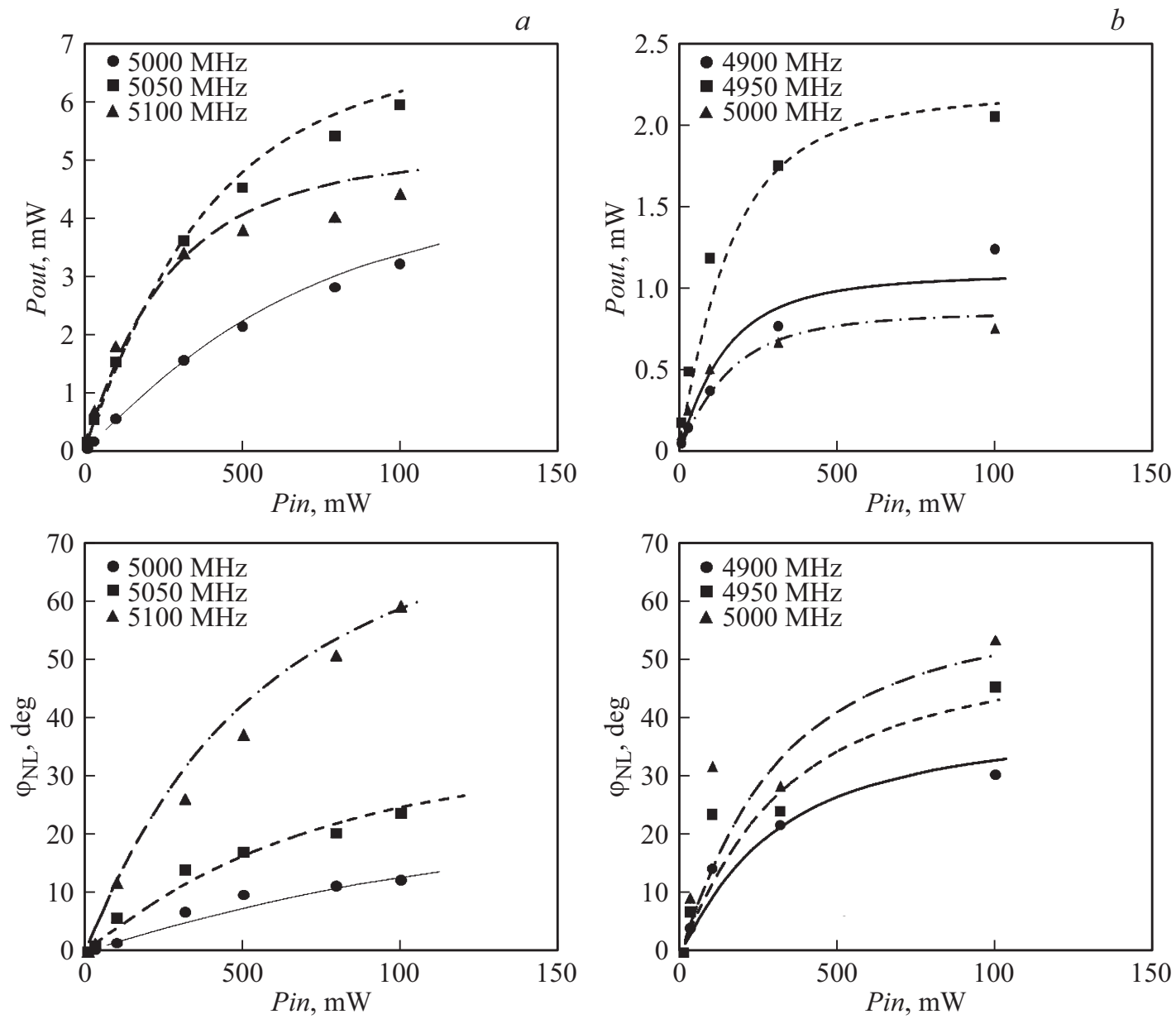

Рис. 3. Зависимости выходной мощности (сверху) и нелинейного фазового сдвига (снизу) СВЧ-сигнала от его входной мощности, полученные для пленок двух толщин: $L_{1}=5.2 \mu \mathrm{m}(a) ; L_{2}=3.1 \mu \mathrm{m}(b)$. Значками показаны результаты измерений, а линиями результаты расчета.

для обоих волноводов совпадали и составляли $7.5 \mathrm{~dB}$. Максимум обратных потерь составлял $11 \mathrm{~dB}$ для пленки толщиной $L_{1}$ и $9 \mathrm{~dB}$ для пленки толщиной $L_{2}$.

При сравнительно низком уровне мощности (не более $0 \mathrm{dBm})$ CВ распространялись в линейном режиме. В таком режиме вносимые потери не зависели от уровня мощности. Увеличение мощности входного сигнала выше $0 \mathrm{dBm}$ приводило к плавному росту вносимых потерь. Так, для волновода СВ толщиной $L_{1}$ при увеличении мощности до $15 \mathrm{dBm}$ потери на частоте $5050 \mathrm{MHz}$ возрастали на $2 \mathrm{~dB}$. Для волновода толщиной $L_{2}$ при увеличении мощности до $15 \mathrm{dBm}$ потери на частоте $4950 \mathrm{MHz}$ возрастали на $5 \mathrm{~dB}$. Такое поведение обусловлено нелинейными затуханием СВ. Из рис. 2 видно, что эффект нелинейного затухания сильнее выражен для более тонкой пленки. Это объясняется более высокой плотностью мощности СВ в пленке меньшей толщины. В нелинейном режиме работы устройства при увеличении мощности входного сигнала также наблюдался незначительный спад обратных потерь. Это было учтено при расчете характеристик распространения интенсивных CB.
Рассмотрим результаты, полученные для трех частот из полосы пропускания макетов. На рис. 3 значками представлены экспериментальные значения выходной мощности $P_{\text {out }}$ и нелинейного фазового сдвига СВЧ-сигнала $\varphi_{N L}$ в зависимости от входной СВЧмощности $P_{i n}$, измеренные для трех частот, указанных на рисунке. Значение $\varphi_{N L}$ определялось как разность фаз на выходе устройства относительно линейного режима распространения СВ (при $P_{\text {in }}=0 \mathrm{dBm}$ ). Анализ результатов показывает, что при увеличении мощности входного сигнала эффект нелинейного затухания приводит к увеличению вносимых потерь и насыщению выходной мощности устройства, причем для тонкой пленки насыщение наступает раньше. При увеличении мощности входного сигнала, кроме увеличения вносимых потерь, происходит изменение фазового набега для всех частот: вначале наблюдается небольшой линейный участок, на котором влияние нелинейного затухания мало, затем наступает ограничение нелинейного сдвига фазы, обусловленное влиянием эффекта нелинейного затухания. С ростом частоты нелинейный сдвиг фазы возрастал. 
Полученные экспериментальные результаты были сопоставлены с имеющейся теорией [11]. На первом этапе была проведена характеризация исследуемой структуры. По фазочастотным характеристикам, измеренным при мощности подаваемого сигнала $P_{i n}=0 \mathrm{dBm}$, были определены дисперсионные характеристики $\omega(k)$ для $\mathrm{CB}$, распространяющихся в экранированной ферритовой пленке в линейном режиме. Детальный анализ экспериментальных данных показал, что дисперсия $\mathrm{CB}$ хорошо описывается формулой для структуры феррит-диэлектрик-металл [17]:

$$
\begin{aligned}
& \left(1+2 \frac{\omega_{H}}{\omega_{M}}+2 \frac{\omega}{\omega_{M}}\right) \\
& \times \frac{\omega_{M}+\left(\omega_{H}-\omega\right)(1+\operatorname{th}(k t))}{\omega_{M}+\left(\omega_{H}+\omega\right)(1-\operatorname{th}(k t))}=\exp (-2 k L),
\end{aligned}
$$

где $\omega_{H}=2 \pi|\gamma| H_{0} ;|\gamma|=2.8 \mathrm{MHz} / \mathrm{Oe}-$ гиромагнитное отношение; $\omega_{M}=2 \pi|\gamma| 4 \pi M_{S} ; t-$ толщина воздушной прослойки между ферритом и металлическим экраном, возникающей из-за не идеального прилегания ферритовой пленки к металлизированной подложке; $\omega=2 \pi f-$ циклическая частота СB; $k$ - волновое число СВ. Расчеты по формуле (1) показали, что в экспериментальной конструкции $t=20 \mu \mathrm{m}$.

Нелинейное затухание и нелинейный фазовый набег СВ были исследованы теоретически с использованием феноменологической модели нелинейного распространения $\mathrm{CB}$, описываемой эволюционным уравнением Ландау-Гинзбурга [11]:

$$
\frac{\partial u}{\partial t}+V_{g} \frac{\partial u}{\partial z}+\left(v_{1}+i N\right)|u|^{2} u+v_{2}|u|^{4}=-\eta u
$$

где $|u|^{2}=m^{2} / \sqrt{ } 2\left(4 \pi M_{S}\right)^{2},|u|-$ нормированная амплитуда $\mathrm{CB} ; m-$ амплитуда $\mathrm{CB} ; V_{g}=\partial \omega / \partial k-$ групповая скорость $\mathrm{CB} ; N=\partial \omega / \partial|u|^{2}$ - нелинейный коэффициент $\mathrm{CB} ; \eta=2 \pi|\gamma| \Delta H$ - декремент линейного временного затухания $\mathrm{CB} ; v_{1}$ и $v_{2}$ - декременты нелинейного затухания третьего и пятого порядков.

Для расчета теоретических характеристик экспериментального макета была использована модель, аналогичная [6]. Коэффициент передачи устройства по мощности определялся как

$$
H_{p}\left(\omega, P_{i n}\right)=H_{e x c}(\omega) H_{S W}\left(\omega, P_{i n}\right) H_{r e c}(\omega),
$$

где $H_{e x c}$ и $H_{r e c}-$ потери на возбуждение и прием $\mathrm{CB}$ в пленке соответственно; $H_{S W}=P_{S W 0} / P_{S W d}-$ затухание $\mathrm{CB} ; P_{S W 0}-$ входная мощность $\mathrm{CB} ; P_{S W d}-$ мощность CB, дошедшая до приемной антенны. Фазовый сдвиг СВЧ-сигнала в макете рассчитывали по формуле

$$
\varphi\left(\omega, P_{i n}\right)=\varphi_{\text {exc }}(\omega)+\varphi_{S W}\left(\omega, P_{i n}\right)+\varphi_{r e c}(\omega),
$$

где, $\varphi_{\text {exc }}$ и $\varphi_{\text {rec }}$ - фазовые сдвиги СВЧ-сигнала, вызванные процессами возбуждения и приема СВ соответственно; $\varphi_{S W}\left(\omega, P_{i n}\right)-$ фазовый набег $\mathrm{CB}$ в пленке.
Коэффициенты нелинейного затухания СВ

\begin{tabular}{c|c|c|c|c|c|c|c}
\hline \multicolumn{4}{c|}{$L=5.2 \mu \mathrm{m}$} & \multicolumn{4}{c}{$L=3.1 \mu \mathrm{m}$} \\
\hline$f$, & $\begin{array}{c}k, \\
\mathrm{MHz}\end{array}$ & $\begin{array}{c}v_{1}, \\
1 / \mathrm{cm}\end{array}$ & $\begin{array}{c}v_{2}, \\
1 / \mathrm{ns}\end{array}$ & $\begin{array}{c}f, \\
\mathrm{MHz}\end{array}$ & $\begin{array}{c}k, \\
1 / \mathrm{cm}\end{array}$ & $\begin{array}{c}v_{1}, \\
1 / \mathrm{ns}\end{array}$ & $\begin{array}{c}v_{2}, \\
1 / \mathrm{ps}\end{array}$ \\
\hline 5000 & 29 & 6.3 & 5.9 & 4900 & 33 & 5.8 & 4.2 \\
5050 & 50 & 4,8 & 4.1 & 4950 & 66 & 3.9 & 3.5 \\
5100 & 77 & 4.0 & 1.2 & 5000 & 106 & 2.6 & 3.1
\end{tabular}

В свою очередь $\varphi_{S W}\left(\omega, P_{i n}\right)$ записывался как

$$
\varphi_{S W}\left(\omega, P_{i n}\right)=\varphi_{L}(\omega)+\varphi_{N L}\left(\omega, P_{i n}\right),
$$

где $\varphi_{L}(\omega)=k(\omega) d-$ линейный фазовый набег $\mathrm{CB}$, $\varphi_{N L}\left(\omega, P_{i n}\right)$ - добавка к линейному фазовому набегу, обусловленная нелинейностью СВ.

Анализ результатов измерений показал, что $\varphi_{\text {exc }}$ и $\varphi_{\text {rec }}$ практически не зависят от мощности входного сигнала. Кроме того, известно, что $\varphi_{\text {exc }}=-\varphi_{\text {rec }}$. Поэтому выражение (4) принимает вид $\varphi\left(\omega, P_{i n}\right)=\varphi_{S W}\left(\omega, P_{i n}\right)$.

Используя описанную модель, были рассчитаны зависимости $P_{\text {out }}\left(P_{i n}\right)$ и $\varphi_{N L}\left(P_{i n}\right)$, которые показаны линиями на рис. 3. Характеристики $H_{S W}\left(\omega, P_{i n}\right)$ и $\varphi_{N L}\left(\omega, P_{i n}\right)$ были рассчитаны с использованием решений уравнения (2), описанных в [11]. Входящие в уравнение (2) групповая скорость СВ $V_{g}$, и нелинейный коэффициент $N$ были численно определены из дисперсионного уравнения (1) для рассматриваемой структуры. Для сопоставления с экспериментальными данными в качестве подгоночного параметра использован коэффициент пропорциональности между мощностью СВ и квадратом нормированной амплитуды СВ. Коэффициенты нелинейного затухания феноменологической модели $v_{1}$ и $v_{2}$ были найдены из условия наилучшего совпадения между экспериментальными и теоретическими данными. Полученные значения $v_{1}$ и $v_{2}$ для соответствующих значений частот и волновых чисел приведены в таблице.

Сопоставление полученных результатов с ранее известными позволяет сделать следующие выводы. Нелинейный фазовый сдвиг в экранированной ферритовой пленке слабо выражен в отличие от свободной (неэкранированной) пленки. Это можно объяснить следующим образом. Нелинейный фазовый набег СВ можно приближенно оценить по формуле:

$$
\varphi_{N L} \approx \Delta k_{N L} \cdot d=\left(\Delta \omega_{N L} / V_{g}\right) \cdot d .
$$

Из формулы (6) видно, что высокая групповая скорость СВ в экранированных пленках приводит к меньшему нелинейному фазовому сдвигу. Эффект нелинейного затухания выражен так же ярко, как и в случае неэкранированной пленки.

Из проведенного исследования следует, что экранированные ферритовые пленки могут ограничивать мощность СВЧ-сигнала, переносимого спиновыми волнами. 
С уменьшением толщины пленки уровень ограничения мощности уменьшается. Изменяя величину поля подмагничивания, возможно выбирать частоты, на которых происходит ограничение мощности. Таким образом, экранированные ферритовые пленки могут применяться для построения различных нелинейных устройств обработки СВЧ-сигналов. В качестве примера можно назвать ограничители мощности.

Авторы выражают благодарность Б.А. Калиникосу за обсуждение работы.

Экспериментальная часть работы выполнена при частичной поддержке Российского научного фонда (грант № 16-12-10440). Теоретическая часть работы выполнена при поддержке госзадания Минобрнауки РФ.

\section{Список литературы}

[1] Исхак У.С. // ТИИЭР тематический выпуск. 1988. Т. 76. № 2. С. 86-104.

[2] Калиникос Б.А, Устинов А.Б., Баруздин С.А. Спинволновые устройства и эхо-процессоры. Монография / Под ред. В.Н. Ушакова. М.: Радиотехника, 2013.

[3] Никитов С.А. // УФН. 2015. Т. 185. С. 1099-1128.

[4] Дмитриев В.Ф., Калиникос Б.А. // Изв. вузов. Физика.1988. № 11. С. 24-53.

[5] Дмитриев В.Ф. // Радиотехника и электроника. 1990. Т. 35. № 9. C. $1821-1828$.

[6] Ustinov A.B., Kalinikos B.A. // Appl. Phys. Lett. 2008. Vol. 93. P. 102504.

[7] Kuanr B.K., Anderson N.R., Celinski Z.J., Camley R.E. // IEEE Magnet. Lett. 2015. Vol. 6. P. 3500304.

[8] Hansen U.H., Demidov V.E., Demokritov S.O. // Appl. Phys. Lett. 2009. Vol. 94. P. 252502.

[9] Устинов А.Б., Калиникос Б.А. // Письма в ЖТФ. 2001. т. 27. Вып. 10. С. 20-25.

[10] Vasiliev S.V., Kruglyak V.V., Sokolovskii M.L., Kuchko A.N. // J. Appl. Phys. 2007. Vol. 101. P. 113919.

[11] Ustinov A.B., Kalinikos B.A. // Appl. Phys. Lett. 2006. Vol. 89. P. 172511.

[12] Sadovnikov A.V., Odintsov S.A., Beginin E.N. et al. // Phys. Rev. B. 2017. Vol. 96. P. 144428.

[13] Кондрашов А.В. // Письма в ЖТФ. 2016. Т. 42. Вып. 17. C. 20-27.

[14] Гришин С.В., Гришин В.С., Романенко Д.В., Шараевский Ю.П. // Письма в ЖТФ. 2014. Т. 40. Вып. 19. C. 51-59.

[15] Scott M.M., Patton C.E., Kostylev M.P, Kalinikos B.A. // J. Appl. Phys. 2004. Vol. 95. N 11. P. 6294.

[16] Гуревич А.Г., Мелков Г.А. Магнитные колебания и волны. М.: Физматлит, 1994. 464 с.

[17] Bongianni W.L. // J. Appl. Phys. 1972. Vol. 43. P. 2541-2544. 